PANORAMA

ISSN: 1909-7433

ISSN: 2145-308X

ednorman@poligran.edu.co

Politécnico Grancolombiano

Colombia

\title{
OBJETO VIRTUAL DE APRENDIZAJE PARA DESARROLLAR LAS HABILIDADES NUMÉRICAS: UNA EXPERIENCIA CON ESTUDIANTES DE EDUCACIÓN BÁSICA
}

Albarracín Villamizar, Carmen Zulay; Hernández Suárez, César Augusto; Rojas Suárez, Jhan Piero OBJETO VIRTUAL DE APRENDIZAJE PARA DESARROLLAR LAS HABILIDADES NUMÉRICAS: UNA EXPERIENCIA CON ESTUDIANTES DE EDUCACION BÁSICA

PANORAMA, vol. 14, núm. 26, 2020

Politécnico Grancolombiano, Colombia

Disponible en: http://www.redalyc.org/articulo.oa?id=343963784007

DOI: https://doi.org/10.15765/pnrm.v14i26.1486.

Esta obra está bajo una Licencia Creative Commons Atribución-NoComercial-SinDerivar 4.0 Internacional. 


\title{
APRENDIZAJE PARA \\ DESARROLLAR LAS HABILIDADES NUMÉRICAS: UNA EXPERIENCIA CON ESTUDIANTES DE EDUCACIÓN BÁSICA
}

\author{
VIRTUAL LEARNING OBJECT FOR DEVELOPING \\ NUMERICAL SKILLS: AN EXPERIENCE WITH BASIC \\ EDUCATION STUDENTS \\ OBJECTO DE APRENDIZAGEM VIRTUAL PARA \\ O DESENVOLVIMENTO DE COMPETÊNCIAS \\ NUMÉRICAS: UMA EXPERIÊNCIA COM \\ ESTUDANTES DO ENSINO BÁSICO
}

PANORAMA, vol. 14, núm. 26, 2020

Politécnico Grancolombiano, Colombia

Recepción: 26 Julio 2018

Aprobación: 05 Febrero 2020

DOI: https://doi.org/10.15765/ pnrm.v14i26.1486.

Redalyc: http://www.redalyc.org/ articulo.oa?id=343963784007

\author{
Carmen Zulay Albarracín Villamizar \\ carmenzulayav@ufps.edu.co \\ Universidad Francisco de Paula Santander, Colombia \\ César Augusto Hernández Suárez cesaraugusto@ufps.edu.co \\ Universidad Francisco de Paula Santander, Colombia \\ Jhan Piero Rojas Suárez jhanpierorojas@ufps.edu.co \\ Universidad Francisco de Paula Santander, Colombia
}

Resumen: El presente reporte tiene como objetivo elaborar un objeto virtual de aprendizaje (OVA), para desarrollar las habilidades numéricas por medio de las operaciones básicas de matemática. La metodología propuesta es aplicada. Se toma el modelo ADDIE para la construcción del OVA. La técnica y el instrumento serán un cuestionario tipo Likert. La evaluación de la calidad del OVA será mediante un equipo evaluador, que emitirá su juicio sobre el carácter didáctico y tecnológico del OVA. Los resultados y el análisis se mostrarán en frecuencias simples para los ítems y promedios de medias para la evaluación del OVA en su carácter didáctico y tecnológico.

Palabras clave: Cursos de educación, matemáticas, sistemas de gestión del aprendizaje, tecnología educativa, virtualización de plataformas.

Abstract: The objective of this research is to develop a virtual learning object to strengthen numerical skills through the basic mathematical operations. The proposed methodology is applied. The ADDIE model is used for the construction of the OVA. The technique and the instrument will be a Likert type questionnaire. To evaluate the quality of the OVA through an evaluation team, which will give their opinion on the didactic and technological character of the OVA. The results and the analysis will be shown in simple frequencies for the items and averages of means for the evaluation of the OVA in its didactic and technological character.

Keywords: Education courses, educational technology, learning management systems, mathematics, platform virtualization.

Resumo: Este relatório visa desenvolver um objecto de aprendizagem virtual (OVA) para desenvolver competências numéricas através de operações matemáticas básicas. A 
metodologia proposta é aplicada. O modelo ADDIE é utilizado para a construção do OVA. A técnica e o instrumento serão um questionário do tipo Likert. A avaliação da qualidade do OVA será feita através de uma equipa de avaliação, que dará o seu parecer sobre o carácter didáctico e tecnológico do OVA. Os resultados e análises serão apresentados em frequências simples para os artigos e médias para a avaliação do OVA no seu carácter didáctico e tecnológico.

Palavras-chave: Cursos de educação, matemática, sistemas de gestão de aprendizagem, tecnologia educacional, virtualização de plataform.

\section{INTRODUCCIÓN}

En las observaciones y los análisis realizados en diferentes instituciones educativas de Colombia se evidencian la falta de comprensión e interpretación que manifiestan los estudiantes acerca de diferentes contenidos relacionados con las cuatro operaciones; dicen no tener claros los conceptos básicos. Por esto cabría preguntarse si estas dificultades pudieran ser causantes de los resultados de bajo rendimiento obtenidos por los estudiantes en las pruebas Saber, tanto en grados tercero, como quinto o noveno. Un análisis descriptivo de las pruebas Saber $3^{\circ}, 5^{\circ}$ y $9^{\circ}$ (de los años 2009, 2012 y 2013), y Saber 11 (del año 2014), en Bogotá, Colombia, recomienda que en Matemáticas de tercero es importante fortalecer aspectos relacionados con la suma y multiplicación; de igual manera, argumenta que en Matemáticas para grado $5^{\circ}$ será importante trabajar en las combinaciones de números, construcción de planos cartesianos, representación de datos y formulación de problemas (Secretaría de Educación del Distrito, 2014).

De igual manera, algunas de las limitaciones que presentan los estudiantes en la comprensión de la matemática radica en el tipo de aprendizaje, junto al miedo que históricamente se ha inculcado sobre la misma, que para Núñez et al. (2006), afirman que son muchos los escolares que perciben las matemáticas como un conocimiento intrínsecamente complejo que genera sentimientos de ansiedad e intranquilidad, constituyendo una de las causas más frecuentes de frustraciones y actitudes negativas; por esto se puede decir que los estudiantes mantienen un temor generalizado frente a ellas durante su educación básica, que pueden influir en los resultados futuros de cada estudiante, generando desmotivación hacia la misma.

Por otra parte, también se da importancia a metodologías tradicionales, al desarrollo de habilidades mecanicistas, repetitivas y de memorización en la resolución de operaciones, lo que trae como consecuencia la falta de relación entre los conceptos, uso de operaciones básicas, aplicación y operacionalización en su cotidianidad. Igualmente, se acusa la falta de estrategias en las nuevas formas, espacios y tiempos para la construcción del conocimiento mediante el aprendizaje activo con Tecnologías de Información y Comunicación (de ahora en adelante, TIC).

En este sentido, las tecnologías inciden drásticamente en los procesos y servicios de cualquier organización, incluidas las educativas, y la falta de ellas actualmente pueden generar desinterés entre los estudiantes, porque según Prensky (2010), estos son nativos digitales que piensan y 
procesan información distinta a sus predecesores, escenario que afectaría alcanzar metas propuestas, adquisición de habilidades numéricas de los estudiantes, así como un desaprovechamiento de las TIC para llegar a los estudiantes con mayor grado de asertividad en el hecho educativo.

Por ello, la gran cantidad de políticas adoptada en los últimos años por los gobiernos de Colombia, a través del Ministerio de Educación Nacional (en adelante, MEN), que promueven el diseño de las estrategias didácticas mediante la implementación, utilización e integración de las TIC en la escuela para que los estudiantes adquieran conocimiento de manera dinámica y autónoma, especialmente en matemáticas (Arévalo y Gamboa, 2015). Según Gil (2002), por medio de las TIC se proveen de nuevas formas de percibir el mundo, y el sujeto mismo.

Sin embargo, hace falta la construcción y elaboración de objetos virtuales de aprendizaje para que sean integrados en diferentes ambientes digitales de aprendizaje, con el fin de desarrollar las habilidades numéricas de los estudiantes, convirtiéndose en herramientas socioeducativas que facilitan la comunicación de información y construcción del conocimiento para satisfacer necesidades en términos educativos, y proporcionar a cada cual el tipo de ayuda específica que demande para alcanzar los estándares de competencia propuestos por el MEN.

En los nuevos espacios de aprendizaje se organizan procesos de enseñanza y aprendizaje que implican el uso tecnologías donde se crean situaciones educativas centradas en los estudiantes para fomentar el autoaprendizaje, así como el pensamiento crítico, entre otros, por medio de objetos de aprendizaje (OA) u objetos virtuales de aprendizaje (OVA), que proporcionan interfaces de trabajo gráfico con los que los discentes interactúan y construyen sus aprendizajes, elementos que permiten mejorar su desempeño en el área de aplicación, aunque para Gómez y Ramírez (2016), los ambientes de aprendizaje, inclusive los mediados por tecnología, requieren reajustes en las praxis pedagógicas, para que los discentes tengan conocimientos previos óptimos.

Entonces, no cabe duda de que la interacción del estudiante con el material didáctico (OVA) es un factor esencial en el proceso de enseñanza y aprendizaje. En especial, las habilidades numéricas en matemáticas sobre las cuatro operaciones básicas. En este sentido, para Beltrán (1993), el aprendizaje se produce como resultado de la práctica. Que en educación podemos entender la práctica como una praxis que implica conocimiento para conseguir determinados fines. La práctica es el saber hacer (Clemente, 2007). Por tanto, ocurre que cuando una persona analiza alguna actividad en la que ha tomado parte, obtiene ese conocimiento.

Este contexto del uso de las TIC y de los objetos virtuales de aprendizaje no solo permite que los estudiantes aprendan en la escuela, sino que los estudiantes o sus representantes planifiquen el uso del tiempo de estudio y establezcan posibilidades de desarrollar actividades propuestas. Igualmente, pueden comunicarse, de manera síncrona o asíncronamente, con sus pares o docentes para resolver problemas, sin importar el horario 
ni la distancia geográfica que los separe, siempre y cuando el OVA esté dispuesto en plataformas online que permitan dicha interacción.

La labor educativa exige actualmente que los docentes desarrollen múltiples competencias vinculadas con la capacidad de diseñar experiencias de aprendizaje significativas, en las que los estudiantes sean el centro del proceso de enseñanza-aprendizaje. En este panorama, la utilización de las TIC y la implementación de la cultura digital dentro del proceso educativo se hacen indispensables de acuerdo con las necesidades de los estudiantes del siglo XXI (Hernández, Arévalo, \& Gamboa, 2016).

De acuerdo con lo anterior, se pueden mencionar los trabajos de Garzón (2013), presentando la sistematización de la experiencia de construcción de una OVA para el área de Matemáticas; Rojas (2015), quien diseñó y elaboró un OVA para la enseñanza y el aprendizaje del álgebra en los estudiantes del grado octavo. También están Jaramillo y Quintero (2014), quienes desarrollaron un ambiente virtual de aprendizaje fundamentado en la lúdica, para estimular el pensamiento aleatorio en los estudiantes de grado cuarto y quinto de primaria. Por otra parte, Rodríguez (2014), quien elaboró un OVA que integra el origami para mejorar la comprensión de los conceptos geométricos, dirigido a docentes de matemáticas que quieren fortalecer el trabajo realizado en el aula. De igual manera, Zapata, Estrada y Chaparro (2015), presentaron el diseño y construcción de un objeto virtual de aprendizaje, como estrategia para fortalecer la comprensión del concepto de fracción en los estudiantes de educación básica.

En vista de la importancia de los objetos virtuales de aprendizaje, esta investigación se justifica porque busca que los individuos del hecho educativo adquieran, desarrollen y/o fortalezcan activamente habilidades numéricas donde puedan experimentar actividades significativas, flexibles y personalizadas; eligiendo o seleccionando sus estrategias de acuerdo con su estilo de aprendizaje y experiencias previas. Lo cual contribuye a formar hábitos de estudios para la modalidad e-learning que, en el futuro no muy lejano, constituirán técnicas para generar el éxito académico de nuestros estudiantes de básica primaria, ofreciendo la mejor educación posible para contribuir, en última instancia, al bien de la sociedad colombiana.

En este sentido, este artículo inicialmente realiza una breve descripción teórica del OVA y, finalmente, profundiza en la metodología para el diseño de elaboración en sus diferentes fases: construcción, procedimientos y métodos para desarrollar las habilidades numéricas de las operaciones básicas en estudiantes de educación básica.

\section{Objeto virtual de aprendizaje (OVA).}

Un OVA es definido como un conjunto de recursos digitales que puede ser utilizado en diversos contextos, con un propósito educativo y constituido por al menos tres componentes internos: contenidos, actividades de aprendizaje y elementos de contextualización (Tovar, 2014). Además, el objeto de aprendizaje debe tener una estructura 
de información externa (metadato), para facilitar su almacenamiento, identificación y recuperación.

Por otra parte, los OVA se enmarcan en los propósitos de las herramientas TIC, las cuales están produciendo cambios en las metodologías de enseñanza y aprendizaje, en la forma en que los profesores y estudiantes se relacionan con el conocimiento, y la manera en que interactúan los agentes involucrados en el proceso educativo (Cabrera, 2014). De igual modo, en lugar de ser un objeto definible, es una construcción tecnológica compleja y multifacética, un rompecabezas tecnológico más grande, porque confluyen con lo pedagógico y curricular que se derivan de las prácticas de la tecnología educativa, de la tecnología de la información y comunicación, entre otros.

De lo anteriormente expuesto, es necesario acotar que esta indagación para la elaboración del OVA para desarrollar las habilidades numéricas se fundamenta en el modelo ADDIE, así como en diversas teorías de aprendizaje: teoría de la información, al generar proceso de fragmentación, quedando así la información dividida en pequeñas piezas (Miller, 1956); teoría del conexionismo (Schneider,1987), donde a partir de la práctica se genera aprendizaje, en función de la cantidad de conexiones aprendidas en situaciones encontradas previamente.

De igual manera, se centra en la teoría constructivista de Bruner, para quien el aprendizaje es un proceso activo en el cual los estudiantes construyen nuevas ideas o conceptos basados en su conocimiento actual/ pasado. Además, el estudiante selecciona y transforma la información, construye hipótesis y toma decisiones, basándose en una estructura cognitiva para hacerlo, con lo cual proporciona significado y organización a las experiencias y permitiendo al individuo ir más allá de la información dada (Bruner, 2006).

Lo anterior está en concordancia con lo planteado por Piaget, en relación que lo nuevo se construye siempre a partir de lo adquirido y lo trasciende (Gómez-Granell \& Coll, 1984), porque al presentar la información en un objeto virtual de aprendizaje, con juegos y de manera diferente a la tradicional sería significativo, y según Ausubel (2002), el individuo construye significados, representaciones mentales relativas a los contenidos, y aprender es conocer, comprender el significado, y esto es posible en la medida de que se produce el anclaje del nuevo material como producto de la motivación, necesidades y deseos, a partir de que el "aprendizaje es activo" (Bruner, Vygotsky, Piaget, Ausubel); del mismo modo, tomando en consideración a Vygotsky (1978), quien dice que la cultura juega un papel principal en el desarrollo humano, en el caso de este trabajo, todo lo que engloba la cultura digital actual. De allí, los objetos virtuales de aprendizaje podrían ser una estrategia interactiva para aprender y para enseñar.

\section{Los OVA en los ambientes virtuales de aprendizaje.}

Los docentes que utilizan las TIC como herramienta pedagógica permiten captar la atención de sus estudiantes proporcionando 
aprendizaje significativo, pero a su vez afrontando retos que plantea la tecnología, cuando utilizan los Ambientes Digitales de Aprendizaje (ADA), o sus equivalentes, tales como Ambiente Virtual de Aprendizaje (AVA), o Entornos Virtuales de Aprendizaje (EVA), por medio de un Sistema de Gestión de Aprendizaje (Learning Management System, siglas en inglés, LMS), como son las plataformas virtuales como Moodle (Cocunubo-Suárez, Parra-Valencia, \& Otálora-Luna, 2018; Prada, Hernández, \& Gamboa, 2019). Estas plataformas ofrecen la oportunidad de administrar OVA, que cumplan con estándares como SCORM, además de plantear estructura informacional enfocada al aprendizaje significativo, y que es excelente para evaluar conocimientos de los estudiantes automáticamente, siendo este el responsable de cada interacción con el OVA.

El SCORM (Shareable Content Object Reference Model), se define como un conjunto de especificaciones que proponen un modelo de agregación de contenidos (Content Aggregation Model, CAM), un entorno de tiempo de ejecución (Run-Time Environment, RTE), y la secuenciación y navegación (Sequencing and Navigation, SN), de los contenidos (Hilera \& Hoya, 2010). Igualmente, argumentan que actualmente SCORM es la norma que está teniendo un mayor impacto en la industria, ya que es la que se ha implementado en un mayor número de sistemas como modelo de referencia (Mayorga, Alfonso, \& Escamilla, 2013). Así pues, distintas herramientas de autor como Exelearning permiten construir objetos virtuales de aprendizaje con salida a un paquete de SCORM (1.2 o 2004), que es un archivo XML que contiene toda la información para poner en marcha en un formato compatible con un LMS (Álvarez, 2017).

\section{Habilidad numérica.}

La habilidad es el dominio de las técnicas, y esta puede ser cognoscitiva o práctica; por ejemplo, las habilidades numéricas de las operaciones básicas de matemáticas, que en aritmética corresponde a la suma, resta, multiplicación y división, se adquieren mediante el conocer, la práctica y resolución de ejercicio, con lo cual se desarrolla una reflexión de los sistemas numéricos. En este contexto, se expone que es la comprensión general que tiene una persona sobre los números y las operaciones junto con la habilidad para hacer juicios matemáticos y desarrollar estrategias útiles al manejar números y operaciones (Obando, Vanegas, \& Vásquez, 2006).

Además, se puede inferir que la habilidad numérica se hace visible en la medida en que se desarrollan estrategias útiles al manejar números y operaciones mediante los diferentes tipos (mental, escrito, estimado, aproximado, exacto y mecánico), que constituyen estrategias didácticas del pensamiento numérico que proporcionan la inteligencia que utilizan los individuos en su vida de forma correcta para cumplir procesos de manera adecuada y así trabajar distintos tópicos de la matemática 
(Galeano \& Ortiz, 2008). De allí se deduce que se pueden incluir en un OVA.

\section{MÉTODO}

El presente estudio es una investigación aplicada, que pretende perfeccionar recursos de aplicación del conocimiento ya obtenido mediante la investigación "pura"; cuya pretensión no es la busqueda de verdades irrefutables, sino el hallazgo de utilidad y procesos replicables (Cazau, 2006. Daza-Orozco, CE; Cera-Ochoa, 2018).

Para el análisis de datos se aplicaron herramientas estadísticas. Por ello, una vez recolectada la información esta fue representada en tablas, cuyo análisis se realizó por medio de la estadística descriptiva y hoja de cálculo de Excel. De manera que para el análisis de las dimensiones del OVA se construyó una tabla de interpretación o baremo contentivo de rango, intervalo y categoría para cada uno de los estadísticos descriptivos utilizados.

Modelo ADDIE.

\begin{tabular}{c|c|c}
\hline Rango & Intervalo & Categoría \\
\hline 5 & 5 & Muy buena calidad \\
\hline 4 & $4-4,99$ & Buena calidad \\
\hline 3 & $3-3,99$ & Nivel medio de calidad \\
\hline 2 & $2-2,99$ & Baja calidad \\
\hline 1 & $1-1,99$ & Muy baja calidad \\
\hline
\end{tabular}

\section{Tabla 1.}

Baremo

Fuente. Los autores

Se cuenta con el modelo ADDIE para el proceso de construcción del OVA. El diseño instruccional sirve de guía para sistematizar procesos de desarrollo de acciones formativas (Belloch, 2017). ADDIE es un modelo de diseño instruccional compuesto por cinco (5) fases: Análisis, Diseño, Desarrollo, Implementación y Evaluación. De igual modo, es necesario señalar que dicho modelo tiene carácter global, sirve como marco de trabajo general para el desarrollo de diferentes proyectos, tanto presenciales como virtuales (Muñoz, 2011). 


\section{MODELO ADIDE}

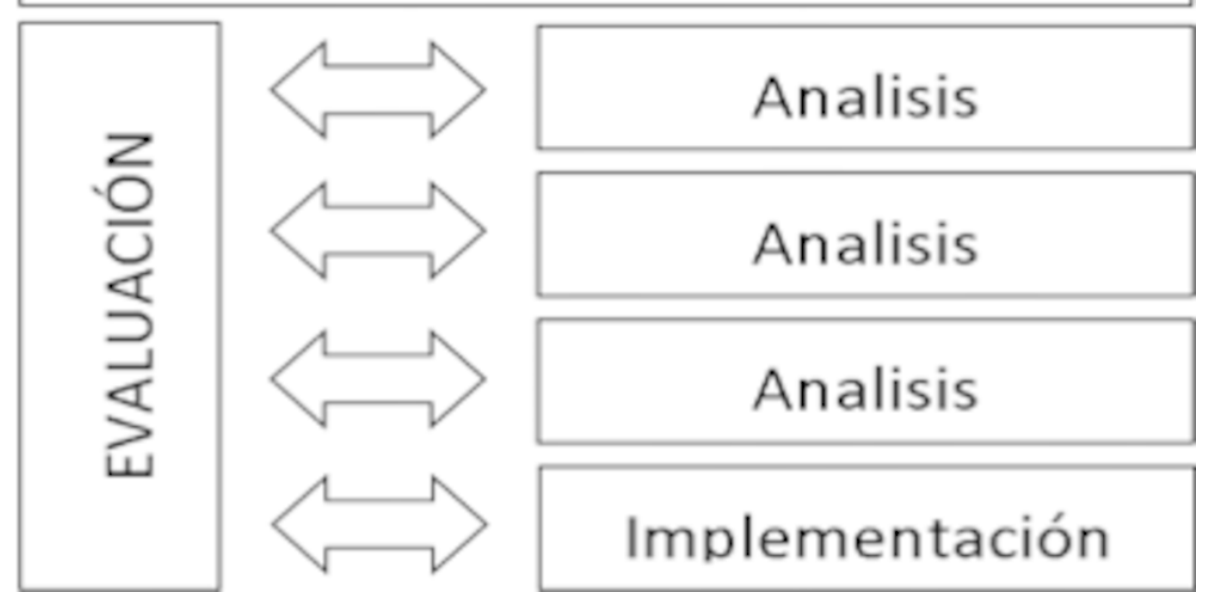

Fig. 1.

Modelo ADDIE.

Fuente. Los autores

De acuerdo con la figura 1, estos pasos pueden seguirse de forma secuencial o bien pueden ser empleados de manera ascendente y simultánea (Muñoz, 2011). Cada elemento de la instrucción es administrado por resultados de aprendizaje, los cuales han sido determinados después de pasar por un análisis de las necesidades del estudiante, por lo que estas fases algunas veces se superponen y pueden estar interrelacionadas, por tanto, proveen una guía dinámica y flexible para el desarrollo efectivo y eficiente de la instrucción (Zapata, Bonfante, \& Suárez, 2013; Bonfante, Lara, \& Chico, 2013). En este mismo orden y dirección, se presenta una adaptación del modelo ADDIE en sus principales fases y evaluaciones; en la figura 2 se muestra el proceso sistemático, planificado y estructurado, que apoya la orientación pedagógica del diseño (Muñoz, 2011).

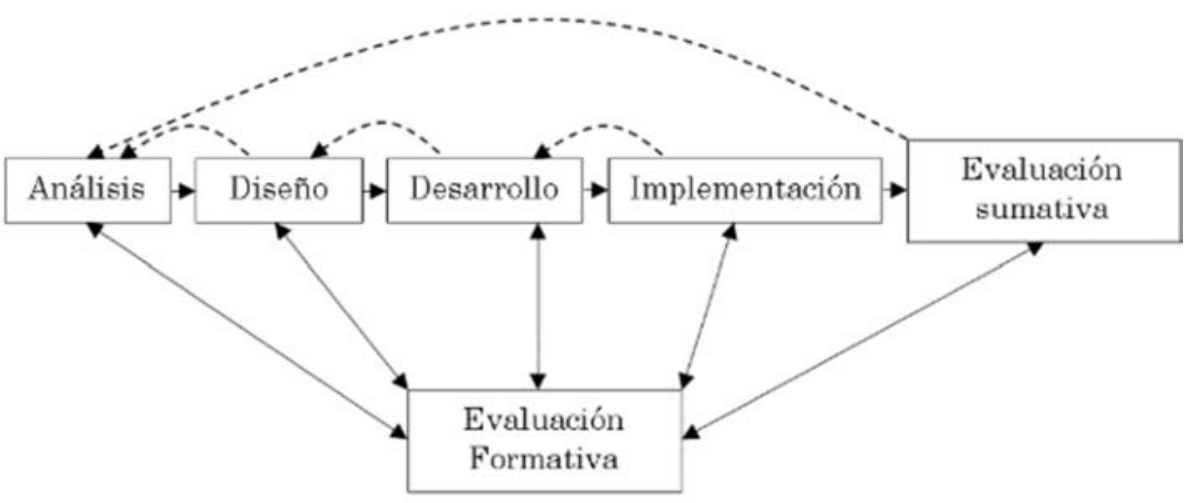

Fig. 2.

Modelo de diseño instruccional ADDIE

Fuente. Los autores 
Análisis.

Según Muñoz (2011), esta es la base de las otras fases del diseño; en ella, el diseñador debe realizar una evaluación de necesidades del entorno (organización), definir el problema, identificar qué causa el problema y buscar posibles soluciones. En este apartado se define el tipo de OVA que se desea construir. En nuestro caso, se desarrolló un OVA de práctica que incluye información y juego educativo para desarrollar las habilidades numéricas de los estudiantes. También se pueden incluir en esta fase técnicas específicas de investigación, tales como: análisis de necesidades, análisis de contexto y análisis de tareas. Las salidas de esta fase generalmente son las metas instruccionales y una lista de tareas a ser instrumentadas (perfil del aprendiz, descripción de obstáculos, necesidades, etc.), las cuales actuarán como inputs para la siguiente fase, es decir, identificación del OVA. En este caso, corresponde describir, en pocas palabras, el contenido temático específico del OVA.

De igual manera, se deben seleccionar las palabras precisas para facilitar su clasificación y búsqueda, además de contener palabras clave, programa, asignatura, docente o responsable, diseñador multimedia. Al mismo tiempo, se identifica los perfiles de quienes pueden ser los posibles usuarios del OVA, por la profundidad y complejidad en su desarrollo conceptual y práctico, y si es necesario, la edad, nivel educativo, contexto, entre otros (Morales, Gutiérrez, \& Ariza, 2016).

Recabada la información durante esta etapa, se comenzó a preparar un bosquejo preliminar del contenido a incluirse en el curso (González, 2011). Se muestra a continuación el contenido preliminar de los temas a enseñar; tomando en consideración la población objetivo, que son estudiantes de educación básica primaria (4 y 5 grado), inclusive hasta el inicio de la educación básica secundaria ( 6 grado). Allí se abordará las cuatro operaciones básicas, con una introducción al álgebra, lo cual los ayudará a afrontar cualquier prueba Saber.

\section{Diseño.}

La fase de diseño implica el manejo de las deducciones obtenidas en la fase de análisis, a fin de concebir una estrategia para el desarrollo del OVA. Durante esta fase se debe delinear el diseño pedagógico, curricular y tecnológico del OVA, para alcanzar las metas educativas determinadas durante la fase de análisis y ampliar los fundamentos educativos (Muñoz, 2011).

\section{Diseño pedagógico.}

Responde a cómo el objeto, en sí mismo, además de ser un recurso didáctico, se convierte en una estrategia para el aprendizaje, donde se definen las clases de actividades que permiten al estudiante desarrollar habilidades con respecto a la temática seleccionada (Morales, Gutiérrez, \& Ariza, 2016). Los conceptos que se encuentran en el OVA deben ser de calidad y de total confiabilidad, revisados y aprobados por un experto en el tema; los conceptos e información deben presentarse 
en forma fragmentada, pero completa, organizada en forma clara y significativa, según los objetivos del currículo, y permitir la navegación del estudiante, guiándose en la comprensión y construcción de su conocimiento (Morales, Gutiérrez, \& Ariza, 2016).

Es decir, se escriben los objetivos de la unidad o módulo, se diseña la evaluación, se escoge los medios y el sistema de hacer llegar la información al alumnado, se determina el enfoque didáctico general, se planifica la formación, decidiendo las partes y el orden del contenido. Se diseñan las actividades para el alumnado y se identifican los recursos necesarios (Muñoz, 2011). Al respecto, los contenidos se deben estructurar y organizar en un sistema de rotulado, navegación y metadatos basado en su relación con el contexto y los usuarios, cumpliendo con los estándares internacionales para OVA como SCORM, asegurando la interoperabilidad, la reusabilidad y la adaptabilidad (Morales, Gutiérrez, \& Ariza, 2016).

Diseño tecnológico.

Se refiere a la selección de las herramientas tecnológicas adecuadas a la creación del OVA, analizando sus posibilidades y limitaciones. Por tanto, constituye el soporte para la integración pedagógica de los contenidos, las actividades de aprendizaje y la evaluación, debido a que se relaciona directamente con la construcción del OVA (Morales, Gutiérrez, \& Ariza, 2016), con el fin de elaborar las diferentes rutas de navegación para que el estudiante o docentes puedan emplear el OVA sin mayor inconveniente.

En el presente proyecto se utilizó la herramienta de autor Exelearning para la construcción del OVA (Garbarini, 2012), además, se utilizó la herramienta Educaplay, donde se crearon otras actividades interactivas que luego fueron integradas al OVA gracias a las bondades que ofrece la web 3.0 para insertar programas, uno dentro de otros, copiando y pegando el código HTML para ese fin (Fernández, 2017; Tupiza, 2018). También se utilizaron herramientas como Animoto, donde se realizaron videos introductorios, y Voki para crear avatar, editándolos en Camtasia para crear video y subirlo a un canal de Youtube y posteriormente ser insertado al OVA. Para el diseño se resalta las rutas de navegación en la interfaz gráfica inicial, con las que cuenta el OVA. 


\begin{tabular}{cl}
\hline Sección & Descripción \\
\hline 1 & Encabezado \\
\hline 2 & Sesión de botones \\
\hline 3 & Banner de la Universidad \\
\hline 4 & Título o temática \\
\hline 5 & Acceso a la información \\
\hline 6 & Barra de navegación \\
\hline
\end{tabular}

Tabla 2.

Interfaz gráfica inicial

Fuente. Los autores
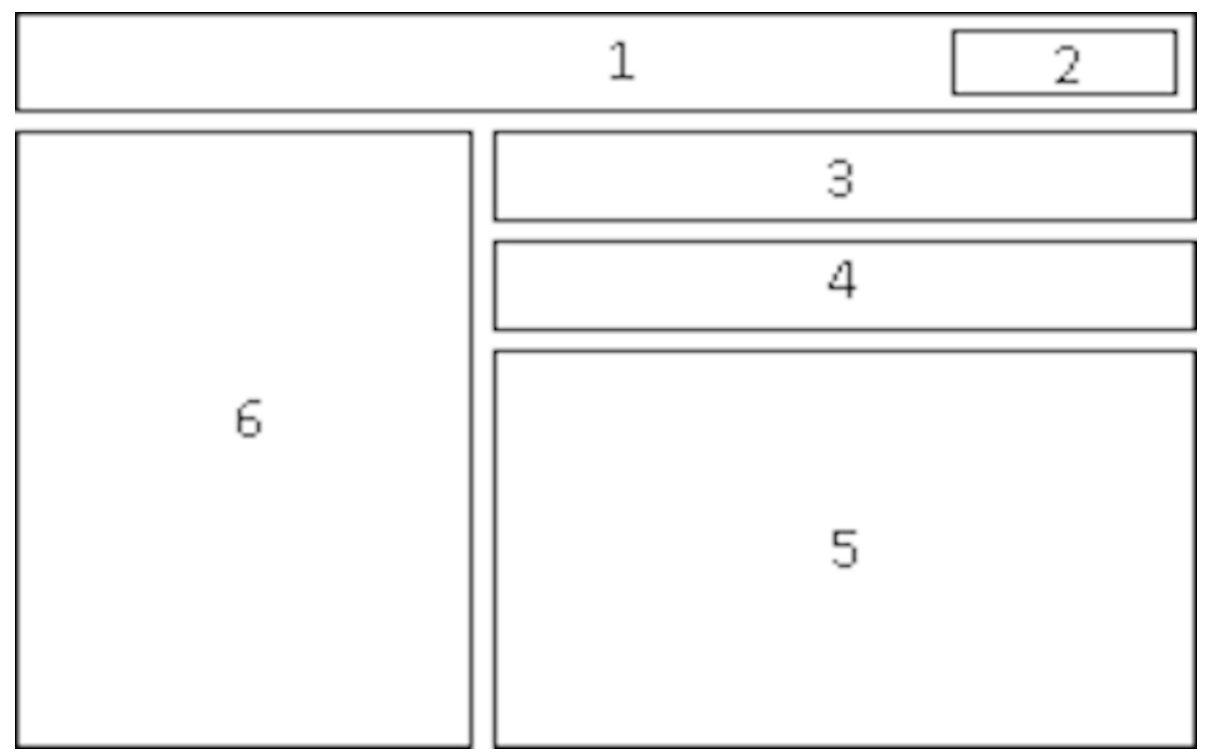

Fig. 3

Interfaz gráfica inicial

Fuente. Los autores

Por otro lado, el plan de actividades de aprendizaje estará conformado por un bosquejo del contenido definido; en este caso, la próxima tarea es preparar un plan del OVA que presenta la secuencia de temas del contenido de las operaciones que corresponden a los objetivos de instrucción. Por tal motivo, se establecen los objetivos y plan de actividades para el OVA, "Las 4 operaciones". 


\begin{tabular}{|c|c|c|}
\hline $\begin{array}{l}\text { Objettivos: } \\
\text { Que el estudiante logre. } \\
\end{array}$ & Por medio de: & $\begin{array}{l}\text { Tipo de actividad en } \\
\text { Exelearning }\end{array}$ \\
\hline $\begin{array}{l}\text { Obtener conocimientos sobre la regla de los } \\
\text { signos }\end{array}$ & $\begin{array}{l}\text { Leer las reglas y ejercicios interactivos prácticos de aplicación de } \\
\text { signos. }\end{array}$ & Rellenar huecos \\
\hline Obtener conocimiento de la tabla de multiplicar & $\begin{array}{l}\text { Actividad interactiva para rellenar los espacios en blanco para } \\
\text { completar la tabla de multiplicar. }\end{array}$ & Rellenar huecos \\
\hline Escritura de cantidades & $\begin{array}{l}\text { Actividad interactiva. Juego del ahorcado, se debe identificar y resolver } \\
\text { las operaciones básicas de matemática en cada planteamiento y el } \\
\text { resultado se debe escribir en letras }\end{array}$ & Juego del ahorcado \\
\hline Obtener conocimiento de suma & Actividad interactiva para resolver sumas simples y llevando & Rellenar huecos \\
\hline $\begin{array}{l}\text { Obtener conocimiento de suma e identificar } \\
\text { unidades y decenas }\end{array}$ & Actividad interactiva. Suma en partes & Rellenar huecos \\
\hline $\begin{array}{l}\text { Obtener conocimiento de resta e identificar } \\
\text { unidades y decenas }\end{array}$ & Actividad interactiva. Resta en partes & Rellenar huecos \\
\hline Repaso de suma y resta & $\begin{array}{l}\text { Actividad interactiva de resolución de ejercicio mentalmente, usando los } \\
\text { trucos de suma en partes y resta en partes }\end{array}$ & Rellenar huecos \\
\hline $\begin{array}{l}\text { Resolver ejercicios simples de la suma, resta y } \\
\text { multiplicación }\end{array}$ & $\begin{array}{l}\text { Actividad interactiva de resolución de ejercicio de sumas, resta y } \\
\text { multiplicación }\end{array}$ & Rellenar huecos \\
\hline $\begin{array}{l}\text { Identificar los tếrminos de la suma, resta y } \\
\text { multiplicacion }\end{array}$ & Lectura de material. & Conocimiento previo \\
\hline $\begin{array}{l}\text { Obtener conocimientos de los términos de las } \\
\text { operaciones }\end{array}$ & Actividad interactiva. Ruleta de conceptos básicos de matemática & $\begin{array}{l}\text { Texto libre. } \\
\text { Insertar actividad interactiva } \\
\text { realizada desde Educaplay }\end{array}$ \\
\hline Obtener conocimiento de multiplicación & Actividad interactiva. Multiplicación a través del juego de dominó & $\begin{array}{l}\text { Texto libre. } \\
\text { Insertar actividad interactiva } \\
\text { realizada desde Educaplay }\end{array}$ \\
\hline Aplicar reglas de signos en la multiplicación. & $\begin{array}{l}\text { Actividad interactiva. Multiplicación de números y signos a través del } \\
\text { juego de domino. }\end{array}$ & Rellenar huecos \\
\hline Aplicar reglas de signos en la division. & $\begin{array}{l}\text { Actividad interactiva. División de números y signos a través del juego de } \\
\text { dominó. }\end{array}$ & $\begin{array}{l}\text { Texto libre. } \\
\text { Insertar actividad interactiva } \\
\text { realizada en Educaplay }\end{array}$ \\
\hline $\begin{array}{l}\text { Identificar expresiones matemáticas con las } \\
\text { operaciones }\end{array}$ & Actividad interactiva. Relaciona las expresiones & $\begin{array}{l}\text { Texto libre. } \\
\text { Insertar actividad interactiva } \\
\text { realizada en Educaplay }\end{array}$ \\
\hline $\begin{array}{l}\text { Obtener conocimientos de expresiones } \\
\text { matemáticas con las operaciones. }\end{array}$ & $\begin{array}{l}\text { Actividad interactiva. Construye la expresión matemática a través de los } \\
\text { términos de las operaciones básicas de matemática. }\end{array}$ & Rellenar huecos \\
\hline Obtener conocimiento sobre ecuaciones & $\begin{array}{l}\text { Actividad interactiva. Construye las ecuaciones, identificando la variable } \\
\text { y resolviendo a través de las operaciones básicas de matemática. }\end{array}$ & Rellenar huecos \\
\hline Identificar los elementos de una potencia. & Actividad interactiva. Potencias y exponentes & Rellenar huecos \\
\hline Resolver ejercicios de potencia & $\begin{array}{l}\text { Actividad interactiva. Resuelve las potencias identificando la base y } \\
\text { exponente y desarrollando la multiplicación }\end{array}$ & Rellenar huecos \\
\hline Resolver problemas verbales & Actividad interactiva. Problemas verbales & Rellenar huecos \\
\hline Identificar la coordenada de un punto. & Lectura material & Conocimiento previo \\
\hline Resolver ejercicios de punto de la coordenada & Actividad interactiva. Hallar puntos de la coordenada. & Rellenar huecos \\
\hline $\begin{array}{l}\text { Identificar y resolver puntos en el plano } \\
\text { cartesiano y sus cuadrantes }\end{array}$ & Actividad interactiva. Puntos en el plano de coordenadas & Rellenar huecos \\
\hline
\end{tabular}

Tabla 3.

Objetivos de aprendizaje, estrategias y tipo de actividad Fuente. Los autores

Los materiales del OVA comprenden textos y recursos multimedios, los mismos contienen imágenes, audio, grabaciones de video. En este apartado se decide qué materiales ya están disponibles, cuáles necesitan ser convertidos de una forma a otra y cuáles necesitan ser creados (González, 2011). Por último, se escoge lo tecnológico que consiste en seleccionar el tipo actividad que proporciona la aplicación Exelearning en función de la estrategia didáctica y el currículo a presentar.

En otro orden de ideas, es necesario acotar que, al aplicar evaluación formativa a la fase de análisis, se determinó que faltaban contenidos para ser abordados, tales como la regla de los signos, conceptos básicos para la resolución de ejercicios que ameriten tal conocimiento; por tal motivo, se agregó a los contenidos del OVA.

\section{Desarrollo.}

La fase de desarrollo se estructura sobre las bases de las fases de análisis y diseño. La intención de esta fase es generar las unidades, módulos y/ o materiales didácticos de las mismas. Durante esta fase se desarrolla la instrucción, todos los medios usados en la instrucción y cualquier documento de apoyo (Duvergel \& Argota, 2017). Entonces, el fin de esta fase es agregar contenido al marco diseñado.

También se escriben los materiales del texto, se graba y editan los recursos multimedia de acuerdo con el diseño realizado en la fase previa. Es primordial que el OVA sea fácil de entender en sus secuencias, 
explicaciones e interpretaciones, por lo que permite dar acceso y motivar a los participantes en su navegación mediante los distintos recursos creados. En esta parte se requiere la confección de instrumentos a utilizar para efectuar los distintos tipos de evaluación creados (González, 2011).

En conclusión, el desarrollo es la creación real (producción), de los contenidos y materiales de aprendizaje basados en la fase de diseño (Belloch, 2017); el mismo tiene que cumplir con lo siguiente (Triana, 2015): (1) el OVA debe presentar los objetivos, así como un diagrama con los temas a tratar; (2) la información presentada en el OVA debe ser clara y legible; (3) la interfaz gráfica del OVA debe contar con logos, escudos y colores alusivos a la universidad, así como la información necesaria para su comprensión y uso, y (4) el OVA debe ser de libre acceso para cualquier estudiante que desee hacer uso de él.

Por otro lado, es necesario señalar que, al aplicar evaluación formativa a la fase de diseño, con relación a la navegabilidad del OVA, donde los contenidos estaban distribuidos en submódulos, se determinó mediante pruebas con estudiantes que esta no permiten que la interfaz gráfica se muestre en su totalidad en el menú, lo cual impide que el discente escoja la temática o tema y estudiar o practicar sus ejercicios planteados para su resolución.

Por tal motivo, se reestructuró la disposición de la temática en el OVA, que a continuación se presenta. De igual manera, se eliminó, del menú "evalúa tu conocimiento", debido a que es un OVA de práctica e información donde los conocimientos son evaluados en cada estrategia dispuesta en la página a navegar en el OVA.

De igual manera, se hicieron ajustes en el diseño del OVA, donde la interfaz gráfica del mismo contara con logos y colores alusivos a la universidad. Igualmente, la información presentada en él debe ser clara y legible, brindando acceso a los diferentes temas establecidos en el contenido programático. Por tal motivo, se realizaron distintas pruebas de múltiples herramientas empleadas con el fin de generar interactividad en el uso del OVA, tanto SCORM como HTML, que fueron los dos formatos de paquetes de exportación del OVA realizados en Exelearning.

A continuación, en la figura 4 se presentan los gráficos con la distribución de los contenidos. 


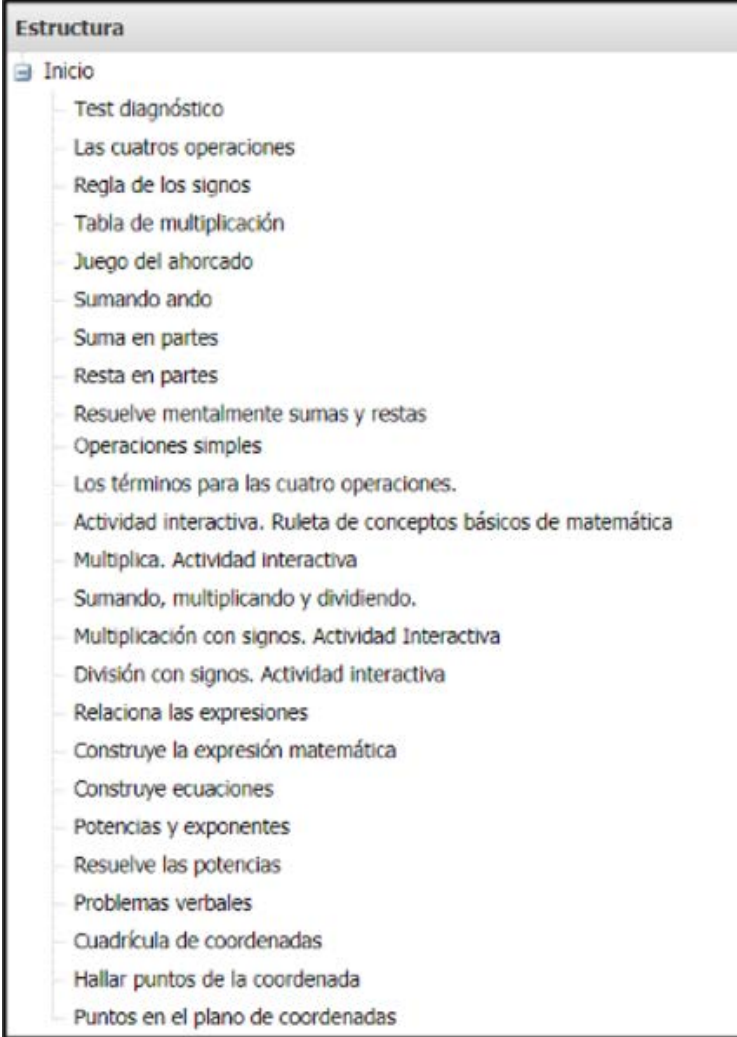

Fig. 4

Estructura de contenidos definidos.

Fuente. Los autores

\section{Construcción y desarrollo del OVA}

Para la construcción y desarrollo del OVA, donde se detalla la navegabilidad de este es necesario tener en cuenta la descripción de los casos de uso del OVA, que a continuación se presentan: 


\begin{tabular}{|c|c|}
\hline \multirow{2}{*}{\multicolumn{2}{|c|}{$\begin{array}{l}\text { Casos de uso OVA } \\
\text { Interacción con el OVA }\end{array}$}} \\
\hline & \\
\hline Actores & Estudiante \\
\hline Función & Usabilidad del OVA \\
\hline Descripción & $\begin{array}{l}\text { Permitir a los estudiantes acceder a los diferentes módulos del OVA, la cual puede estar disponible en una } \\
\text { plataforma LMS (como Moodle) o en su defecto alojada como una página web en internet. }\end{array}$ \\
\hline Sucesos del evento: & $\begin{array}{l}\text { - Visualizar el OVA } \\
\text { - Acceso al OVA } \\
\text { - Acceso a barra de navegación del OVA } \\
\text { - Accturas, videos, actividades evaluadas. }\end{array}$ \\
\hline \multicolumn{2}{|r|}{ Desarrollo del test para diagnóstico } \\
\hline Actores & Estudiante \\
\hline Función & Ingreso al test. \\
\hline Descripción & Test para diagnosticar conocimientos previos del estudiante. \\
\hline Sucesos del evento: & $\begin{array}{l}\text { - Visualizar el test del OVA } \\
\text { - Ecceso al test } \\
\text { - Realimentación del test. }\end{array}$ \\
\hline \multicolumn{2}{|r|}{ Desarrollo de recursos didácticos (acceso a lectura) } \\
\hline Actores & Estudiante \\
\hline Función & Ingreso a lectura \\
\hline Descripción & $\begin{array}{l}\text { El estudiante tiene acceso a la información como lectura, diagramas e imágenes relacionadas a las } \\
\text { operaciones. }\end{array}$ \\
\hline Sucesos del evento: & $\begin{array}{l}\text { - Visualizar lectura del OVA } \\
\text { - Lectura de los temas } \\
\text { Visualización de diagramas }\end{array}$ \\
\hline \multicolumn{2}{|r|}{ Desarrollo de recursos didácticos (actividades) } \\
\hline Actores & Estudiante \\
\hline Función & Ingreso a las actividades \\
\hline Descripción & $\begin{array}{l}\text { El estudiante tiene acceso a la información para gestionar su proceso de autoaprendizaje a través de la } \\
\text { práctica y resolución de ejercicios de las operaciones, el mismo, permite la evaluación en cada temática } \\
\text { abordada. }\end{array}$ \\
\hline Sucesos del evento: & $\begin{array}{l}\text { - Selección de la actividad. } \\
\text { - Desarrollo de la actividad en el OVA }\end{array}$ \\
\hline
\end{tabular}

Tabla 4.

Descripción de los casos de uso del OVA.

Fuente. Los autores

DESARROLLO DEL OVA

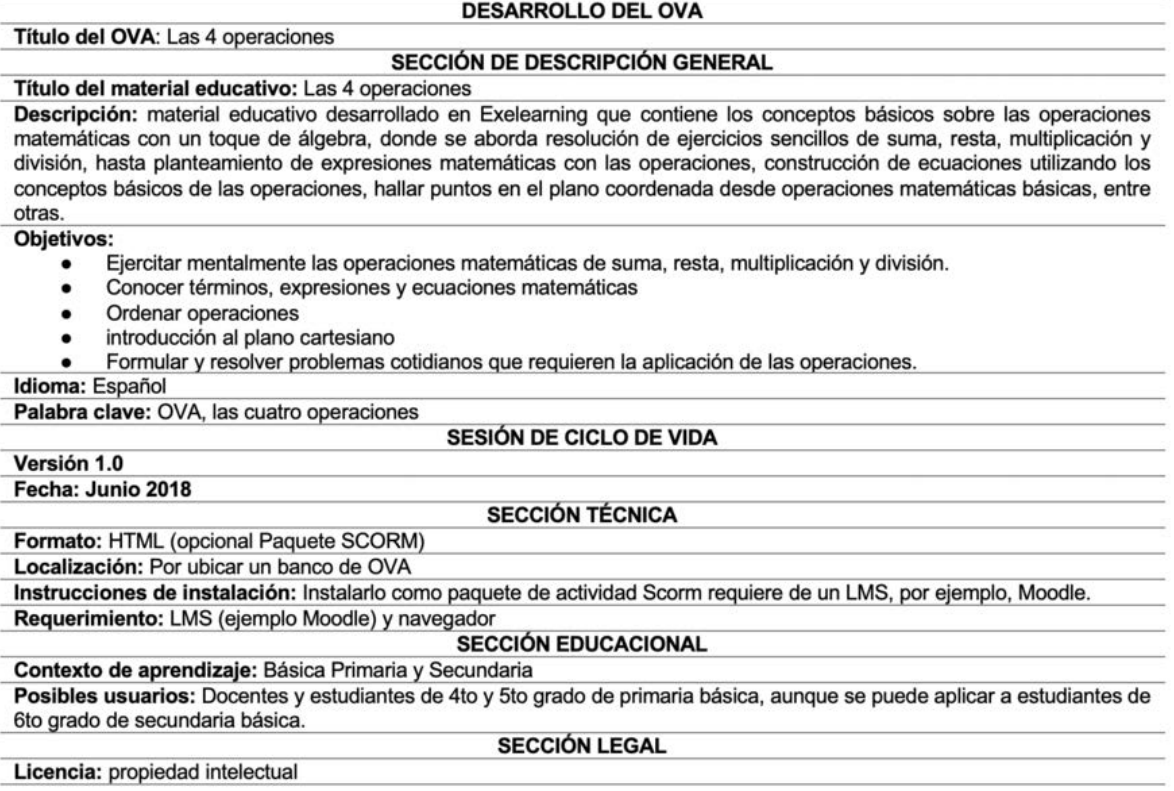

Tabla 5.

Desarrollo del OVA

Fuente. Los autores

\section{Implementación}

La finalidad de esta fase es promover el entendimiento de los materiales por parte de los estudiantes, apoyar su dominio de los objetivos y hacerle un seguimiento a la transferencia de los conocimientos a su actuación 
diaria (Sarmiento, 2007), es decir, ejecución y puesta en práctica de la acción formativa por medio del OVA (Belloch, 2017). Es necesario acotar que se realizó una prueba del OVA, como paquete Scorm 1.2 en un aula virtual en Moodle.

\section{Evaluación}

Aunque se presenta como la quinta fase del modelo del diseño instruccional, las evaluaciones, son un componente integral de cada uno de las cuatro fases anteriores. Al conducir cada fase del diseño instruccional, los procedimientos y actividades pueden ser evaluadas para asegurar que se realicen en la manera más eficaz para asegurar resultados óptimos (Muñoz, 2011; Ceballos, Mejía, \& Botero, 2019).

\section{RESULTADOS}

Según Fernández-Pampillón, Domínguez y Armas (2012), un OVA es de calidad cuando es eficaz, didáctico, produce buenos resultados académicos y tecnológicos; además, argumentan que para valorar efectividad tecnológica y didáctica de los OVA se debe aplicar herramientas de evaluación que se aprovechen de guía como COdA, el cual consta de un formulario con diez criterios de calidad puntuables de uno (1) como mínimo a cinco (5) como máximo. Los cinco primeros criterios son de carácter didáctico, mientras que los otros cinco son tecnológicos, de manera que ambos aspectos tienen el mismo peso. A saber:

\begin{tabular}{cl}
\hline $\begin{array}{c}\text { Objetivos y } \\
\text { coherencia didáctica }\end{array}$ & $\begin{array}{l}\text { Evalúa la definición y coherencia de los objetivos, asi como las especificaciones de uso para } \\
\text { el usuario }\end{array}$ \\
\hline $\begin{array}{c}\text { Calidad de contenidos } \\
\text { Evaluar el contenido del objeto de aprendizaje, destacando la exposición del contenido y el } \\
\text { uso de actividades }\end{array}$ \\
$\begin{array}{c}\text { Capacidad de generar } \\
\text { reflexión }\end{array}$ & $\begin{array}{l}\text { Evalúa el desarrollo de habilidades para la resolución de tareas o actividades para generar } \\
\text { nuevas ideas y buscar nuevos conocimientos en el estudiante }\end{array}$ \\
\hline $\begin{array}{c}\text { Interactividad y } \\
\text { adaptabilidad } \\
\text { Se valora tanto la dinámica de la presentación del contenido en relación con el uso que haga } \\
\text { de usuarios, sean estudiantes o docentes virtual de aprendizaje se adapta a diferentes tipos }\end{array}$ \\
\hline Motivación & $\begin{array}{l}\text { Evalúa si el objeto de aprendizaje es capaz de atraer al estudiante para aprender y adquirir } \\
\text { nuevos conocimientos con propuestas innovadoras y contenidos didácticos }\end{array}$ \\
\hline Formato y diseño & $\begin{array}{l}\text { Este criterio se encarga de evaluar el diseño, los contenidos y la aplicación de multimedia en } \\
\text { el objeto de aprendizaje }\end{array}$ \\
\hline Usabilidad & $\begin{array}{l}\text { Mide la facilidad con la que el usuario interacciona con el objeto de aprendizaje, tanto en el } \\
\text { contenido como en la interfaz }\end{array}$ \\
\hline Accesibilidad & $\begin{array}{l}\text { Califica si el OVA está construido considerando personas con dificultades audiovisuales o } \\
\text { motrices }\end{array}$ \\
\hline Reusabilidad & $\begin{array}{l}\text { Este criterio se refiere a la capacidad y posibilidad de usar el OVA varias veces, a su vez } \\
\text { valora si sus partes sirven para la construcción de nuevos objetos de aprendizaje }\end{array}$ \\
\hline $\begin{array}{c}\text { Evalua la capacidad de emplear el OVA en diferentes entornos, como en aulas virtuales, por } \\
\text { medio de un LMS, entornos web y ordenadores personales }\end{array}$ \\
\hline Interoperabilidad
\end{tabular}

Tabla 6.

Criterios didácticos y tecnológicos para evaluar un OVA

Fuente. Los autores

Por lo anteriormente expuesto, se realizó la evaluación contando con la colaboración de tres expertos (un especialista en educación, un experto en OVA y un metodólogo), como evaluadores del objeto virtual de aprendizaje. De igual manera, presenta un baremo sobre la calidad del OVA con base en las respuestas emitidas por los evaluadores. En consecuencia, los resultados obtenidos se muestran a continuación, para cuyo propósito se realizaron tablas estadísticas de frecuencia y porcentaje, 
de acuerdo con la dimensión didáctica y tecnológica a la cual se refiere, determinando la frecuencia de respuestas para cada ítem, efectuando su valoración en forma porcentual mediante porcentajes absolutos. A continuación, los resultados obtenidos

\begin{tabular}{ccccccccccc}
\hline Items & \multicolumn{2}{c}{$\begin{array}{c}\text { Muy buena } \\
\text { calidad }\end{array}$} & \multicolumn{2}{c}{ Buena calidad } & \multicolumn{2}{c}{$\begin{array}{c}\text { Nivel medio de } \\
\text { calidad }\end{array}$} & \multicolumn{2}{c}{ Baja calidad } & \multicolumn{2}{c}{$\begin{array}{c}\text { Muy baja } \\
\text { calidad }\end{array}$} \\
\cline { 2 - 14 } & Fs & $\%$ & Fs & $\%$ & Fs & $\%$ & Fs & $\%$ & Fs & $\%$ \\
\hline 1 & 0 & $0 \%$ & 3 & $100 \%$ & 0 & $0 \%$ & 0 & $0 \%$ & 0 & $0 \%$ \\
\hline 2 & 1 & $33,3 \%$ & 2 & $66,7 \%$ & 0 & $0 \%$ & 0 & $0 \%$ & 0 & $0 \%$ \\
\hline 3 & 2 & $66,7 \%$ & 1 & $33,3 \%$ & 0 & $0 \%$ & 0 & $0 \%$ & 0 & $0 \%$ \\
\hline 4 & 1 & $33,3 \%$ & 2 & $66,7 \%$ & 0 & $0 \%$ & 0 & $0 \%$ & 0 & $0 \%$ \\
\hline 5 & 2 & $66,7 \%$ & 1 & $33,3 \%$ & 0 & $0 \%$ & 0 & $0 \%$ & 0 & $0 \%$ \\
\hline
\end{tabular}

Tabla 7.

Carácter didáctico del OVA

Fuente. Los autores

La tabla 7 muestra los ítems del 1 al 5, que hacen referencia a criterios de carácter didáctico para evaluar el objeto virtual de aprendizaje. Atendiendo a los resultados observados en los ítems 1, 2, 3, 4 y 5, es preciso señalar que los resultados coinciden con el planteamiento de Zapata, Estrada y Chaparro (2015), en cuanto se deben desarrollar estrategias útiles al manejar números y operaciones a través de los diferentes tipos (mental, escrito, exacto y mecánico), que constituyen estrategias de carácter didáctica del OVA, y que se evidencia la calidad como buena con tendencia a muy buena calidad del OVA por parte de los evaluadores, para desarrollar las habilidades numéricas, al estar en correspondencia con Tovar (2004), cuando dicen que un OVA es un conjunto de recursos digitales que puede ser utilizado en diversos contextos, con un propósito educativo y constituido por al menos tres componentes internos: contenidos, actividades de aprendizaje y elementos de contextualización.

Por todo lo anteriormente expuesto, se infiere que la educación -al estar inmersa en las nuevas tendencias educativas-, se ve obligada a buscar nuevas manera de enseñar y aprender para innovar con tecnología. Los OVA, al estar enmarcados dentro de las TIC, están produciendo cambios en las metodologías de enseñanza y aprendizaje, en la forma en que los profesores y estudiantes se relacionan con el conocimiento y la manera en que interactúan (Cabrera, 2014), de manera coherente con contenidos adecuados y el discente, para generar reflexión y motivación a partir de la interactividad de los mismos.

\begin{tabular}{ccccccccccc} 
Titems & \multicolumn{2}{c}{$\begin{array}{c}\text { Muy buena } \\
\text { calidad }\end{array}$} & \multicolumn{2}{c}{ Buena calidad } & \multicolumn{2}{c}{$\begin{array}{c}\text { Nivel medio de } \\
\text { calidad }\end{array}$} & \multicolumn{2}{c}{ Baja calidad } & \multicolumn{2}{c}{$\begin{array}{c}\text { Muy baja } \\
\text { calidad }\end{array}$} \\
\cline { 2 - 16 } & Fs & $\%$ & Fs & $\%$ & Fs & $\%$ & Fs & $\%$ & Fs & $\%$ \\
\hline 6 & 0 & $0 \%$ & 3 & $100 \%$ & 0 & $0 \%$ & 0 & $0 \%$ & 0 & $0 \%$ \\
\hline 7 & 1 & $33,3 \%$ & 2 & $66,7 \%$ & 0 & $0 \%$ & 0 & $0 \%$ & 0 & $0 \%$ \\
\hline 8 & 1 & $33,3 \%$ & 2 & $66,7 \%$ & 0 & $0 \%$ & 0 & $0 \%$ & 0 & $0 \%$ \\
\hline 9 & 2 & $66,7 \%$ & 1 & $33,3 \%$ & 0 & $0 \%$ & 0 & $0 \%$ & 0 & $0 \%$ \\
\hline 10 & 1 & $33,3 \%$ & 2 & $66,7 \%$ & 0 & $0 \%$ & 0 & $0 \%$ & 0 & $0 \%$ \\
\hline
\end{tabular}

Tabla 8.

Carácter tecnológico del OVA

Los autores

En la tabla 8 se muestran los ítems del 6 al 10, los cuales informan sobre los criterios de carácter tecnológico evaluados en el OVA. Los resultados 
obtenidos de los ítems 6,7,8,9 y 10, evidencian que el OVA es de buena calidad con tendencia a muy buena calidad en su carácter tecnológico. Esto coincide con lo destacado por Callejas, Hernández y Pinzón (2011), donde los objetos de aprendizaje deben ser flexibilidad y reutilizables para ser usado en múltiples contextos educativos, así como adaptado a los diferentes estilos de aprendizaje de los discentes, de igual manera que sea interoperable para integrarse en diferentes plataformas, como en este, en que el OVA fue exportado y probado como un paquete SCORM.

De acuerdo con lo que se ha venido realizando, se puede inferir que al estar inmersos en las nuevas tendencias educativas, un mundo cada día más conectado que aprovecha oportunidades existentes y crea otras nuevas en los estudios mediados con tecnologías, en lo que respecta a lo didáctico y pedagógico del OVA, esto conduce a realizar un análisis en los caracteres del OVA antes mencionado; de allí la Tabla 9.

\begin{tabular}{|c|c|c|c|c|c|c|c|}
\hline Ítem & $\mathrm{x}$ & Carácter & X Carácter & Criterio & OVA & XOVA & Criterio \\
\hline 1 & 4,00 & \multirow{5}{*}{ Didáctico } & \multirow{5}{*}{4,40} & \multirow{5}{*}{$\begin{array}{l}\text { Buena } \\
\text { calidad }\end{array}$} & \multirow{10}{*}{$\begin{array}{l}\text { Evaluación de } \\
\text { calidad }\end{array}$} & \multirow{10}{*}{4,37} & \multirow{10}{*}{$\begin{array}{l}\text { Buena } \\
\text { calidad }\end{array}$} \\
\hline 2 & 4,33 & & & & & & \\
\hline 3 & 4,67 & & & & & & \\
\hline 4 & 4,33 & & & & & & \\
\hline 5 & 4,67 & & & & & & \\
\hline 6 & 4,00 & \multirow{5}{*}{ Tecnológico } & \multirow{5}{*}{4,33} & \multirow{5}{*}{$\begin{array}{l}\text { Buena } \\
\text { calidad }\end{array}$} & & & \\
\hline 7 & 4,33 & & & & & & \\
\hline 8 & 4,33 & & & & & & \\
\hline 9 & 4,67 & & & & & & \\
\hline 10 & 4,67 & & & & & & \\
\hline
\end{tabular}

\section{Tabla 9}

Evaluación de calidad del OVA.

Fuente. Los autores

En los resultados obtenidos se puede visualizar que el carácter didáctico alcanzó el mayor puntaje de promedio de media con 4,4, que ubica al OVA en un criterio de buena calidad pedagógica, seguido por el aspecto tecnológico que promedió 4,33, que igualmente se ubicó en un criterio de bueno en el carácter tecnológico, lo cual benefició el análisis, diseño, desarrollo, implementación y evaluación del OVA.

El promedio general para la dimensión fue de 4,37, que la ubica, de igual manera, en la categoría de buena calidad con tendencia a muy buena calidad en la elaboración del OVA. Por tanto, los resultados evidencian que una guía metodológica en la elaboración, creación y evaluación de OVA, podría dar indicios para la creación fundamentada en el análisis y reflexión del diseño de los OVA.

\section{DISCUSIÓN}

Lo planteado en las bases teóricas proporciona referentes para el análisis, diseño, desarrollo, construcción y evaluación de los objetos virtuales de aprendizaje, que pueden apoyar la práctica pedagógica de los profesores con métodos innovadores que permitan el aprender buscando, el aprender haciendo, el aprender interactuando por medio de un OVA.

Se verificó, con base en los resultados obtenidos, que al hacer un análisis de necesidades e identificación de los requerimientos curriculares, didácticos y tecnológicos, así como la población objetivo, permiten programar contenidos, objetivos y coherencia didáctica de 
buena calidad, siendo esto beneficioso en la elaboración y producción de OVA, herramientas que buscan desarrollar habilidades, destrezas y competencias en los estudiantes en un área determinada de aprendizaje, a partir de instrucciones adecuadas al nivel de conocimiento de los destinatarios, así como relacionar conceptos ya aprendidos con los nuevos conocimientos.

Obviamente, con el fin del aprovechamiento del aprendizaje mediado por las TIC en los procesos educativos para acceder al conocimiento, sin limitaciones de espacio ni tiempo, los OVA pueden presentar información o prácticas esenciales en un área específica del currículo por parte del que aprende como del encargado de impartir los estudios.

Por otra parte, se comprobó que las evaluaciones tanto formativas como sumativas ayudan a los diseñadores de objetos virtuales de aprendizaje a diseñar y crear OVA de buena calidad en su carácter didáctico y tecnológico. Es decir, el diseño y construcción de OVA accesible, interactivo, con estrategias pedagógicas fáciles de usar en la interacción del estudiante con el contenido didáctico para aprender, practicar y fortalecer cualquier área de conocimiento a la cual esté destinado dicho OVA.

Con relación a este caso, para desarrollar las habilidades numéricas a partir de las operaciones básicas de matemática, el equipo evaluador determinó que el OVA es de buena calidad didáctica y tecnológica con tendencia a muy buena calidad en su diseño y creación, porque favorecen la comprensión y asimilación del conocimiento de estas operaciones matemáticas por la forma innovadora y atractiva de los contenidos y/o los procedimientos didácticos que se presentan en el OVA.

\section{REFERENCIAS}

1. Álvarez, E. (2017). La didáctica de la lengua en entornos virtuales de aprendizaje: el caso concreto de la enseñanza-aprendizaje del español como lengua extranjera y la plataforma Eleclips. Revista de Educación a Distancia, (55), 1-20.

2. Arévalo, M., \& Gamboa, A. (2015). Las tecnologías de la información y de la comunicación en el currículo de matemáticas: orientación desde las políticas y proyectos educativos. Panorama, 9(16), 21-30.

3. Ausubel, D. P. (2002). Adquisición y retención del conocimiento. Una perspectiva cognitiva. Barcelona: Paidós.

4. Belloch, C. (2017). Diseño instruccional. Valencia, España: Universidad de Valencia.

5. Beltrán, J. (1993). Procesos, Estrategias y Técnicas de Aprendizaje. Madrid: Editorial.

6. Bonfante, M., Lara, J., \& Chico, M. (2013). Diseño instruccional de objetos de aprendizaje para niños sordos. Desarrollo de la Inteligencia. Hexágono Pedagógico, 4(1), 132-148. DOI: https://doi.org/10.22519/2145888X.3 14

7. Bruner, J. (2006). Actos de significado más allá de la revolución cognitiva. Alianza. 
8. Cabrera, J. (2014). Un Objeto Virtual de Aprendizaje (OVA) para el Movimiento Armónico Simple (M.A.S) y sus Aplicaciones. Entornos, (28), 71-85. DOI: https://doi.org/10.25054/01247905.526

9. Callejas, M., Hernández, E., \& Pinzón, J. (2011). Objetos de aprendizaje, un estado del arte, Entramado, 7(1), 176-189.

10. Cazau, P. (2006). Introducción a la investigación en ciencias sociales. Buenos Aires.

11. Ceballos, O., Mejía, L., \& Botero, J. (2019). Importancia de la medición y evaluación de la usabilidad de un objeto virtual de aprendizaje. Revista Panorama, 13(25), 24 - 37. DOI: http://dx.doi.org/10.15765/pnrm.v13 i25.xxxxxx

12. Clemente, M. (2007). La complejidad de las relaciones teoría-práctica en educación. Teoría de la Educación. Revista Interuniversitaria, 19(1), $25-46$.

13. Cocunubo-Suárez, J., Parra-Valencia, J., \& Otálora-Luna, J. (2018). Propuesta para la evaluación de Entornos Virtuales de Enseñanza Aprendizaje con base en estándares de Usabilidad. TecnoLógicas, 21(41), 135-147.

14. Daza-Orozco, CE; Cera-Ochoa, R. (2018). Escritura con estilo: Guía práctica para publicar científicamente (1st ed.). Fundación Universitaria San Mateo. http://palma.sanmateo.edu.co/index.php/catalogo/series/41 -escritura-con-estilo-guia-practica-para-publicar-cientificamente

15. Duvergel, Y., \& Argota, L. (2017). Metodología para el diseño instruccional en la asignatura fago de la carrera Ingeniería en Ciencias Informáticas. En VII Congreso Virtual Iberoamericano de Calidad en Educación Virtual y a Distancia,EduQ@2017, Mendoza: Editorial Virtual Argentina.

16. Fernández, M. (2017). Herramienta multimedia (Educaplay) como estrategia para el aprendizaje de química general en segundo semestre de la Carrera de Biología, Química y Laboratorio periodo octubre 2016-marzo 2017 (Trabajo de Investigación). Universidad Nacional del Chimborazo, Riobamba, Ecuador.

17. Fernández-Pampillón, A., Domínguez, E., \& Armas, I. (2012). Herramienta de Evaluación de la Calidad de Objetos de Aprendizaje (herramienta COdA). Madrid: Universidad Complutense de Madrid.

18. Galeano, M., \& Ortiz, D. (2008), El cálculo mental como estrategia para desarrollar el pensamiento numérico. Universidad de Antioquia, Medellín.

19. Garbarini, L. (2012). Campus virtual/Manual del docente. Lanús, Argentina: Universidad Nacional de Lanús.

20. Garzón, J. (2013). Objeto virtual de aprendizaje para el área de matemáticas (Trabajo de Maestría). Universidad Pontificia Bolivariana, Medellín, Colombia.

21. Gil, E. (2002). Identidad y nuevas tecnologías: repensando las posibilidades de intervención para la transformación social. Barcelona: Universidad Abierta de Cataluña - UOC.

22. Gómez, C., \& Ramírez, R. (2016). Comunidades de aprendizaje móvil, Mastery Learning y el Problema 2 sigma como estrategias para el conocimiento previo, en la resolución de actividades. Ecomatemático, 8(S1), 25 - 32. DOI: https://doi.org/10.22463/17948231.1376 
23. Gómez-Granell, C., \& Coll, C. (1984). De qué hablamos cuando hablamos de constructivismo. Cuadernos de Pedagogía, (221), 8-10.

24. González, S. (2011). Un modelo blended learning aplicado a las universidades. Caso: Universidad Inca Garcilaso de la Vega (Tesis de maestría), Universidad Inca Garcilaso de la Vega, Lima, Perú.

25. Hernández, C., Arévalo, M., \& Gamboa, A. (2016). Competencias TIC para el desarrollo profesional docente en educación básica. Praxis \& Saber, 7(14), 41-69, DOI: http://dx.doi.org/10.19053/22160159.5217

26. Hilera, J., \& Hoya, R. (2010). Estándares de E-learning: Guía de consulta. Universidad de Alcalá, España.

27. Jaramillo, J., \& Quintero, D. (2014). Desarrollo de un ambiente virtual de aprendizaje fundamentado en la lúdica que estimule el pensamiento aleatorio en los estudiantes de grado cuarto y quinto de primaria de la Institución educativa el Hormiguero (Trabajo de maestría). Universidad Libre, seccional Cali. Cali, Colombia.

28. Mayorga, M., Alfonso, D., \& Escamilla, R. (2013). Los contenidos educativos digitales como apoyo al proceso de aprendizaje en programas virtuales de la Universidad Antonio Nariño. En XIV Encuentro internacional Virtual Educa, Medellín, Colombia.

29. Miller, G. (1956). The magical number seven, plus or minus two: Some limits on our capacity for processing information. Psychological Review, (63), 81-97.

30. Morales, L., Gutiérrez, L., \& Ariza, L. (2016). Guía para el diseño de objetos virtuales de aprendizaje (OVA). Aplicación al proceso enseñanzaaprendizaje del área bajo la curva de cálculo integral. Revista Científica General José María Córdova, 14(8), 127-147.

31. Muñoz, P. (2011). Modelos de diseño instruccional utilizados en ambientes teleformativos. Revista de Investigación Educativa ConeCT@2, 2(2), 29-62.

32. Núñez, J., González-Pienda, J., Paloma, L., González-Pumariega, S., Roces, C., Castejón, L., Solano, P., Bernardo, A., García, D., da Silva, E., Rosário, P., \& Rodrígues, L. (2006), "Las actitudes hacia las Matemáticas: perspectiva evolutiva”. En Actas do VIII Congreso Galaico-Portugués de Psicopedagoxía, pp. 2389-2396.

33. Obando, G., Vanegas, M., \& Vásquez, N. (2006), Módulo 1. Pensamiento Numérico y Sistemas Numéricos. Medellín, Colombia: Editorial Artes y Letras.

34. Prada, R., Hernández, C., \& Gamboa, A. (2019). Usos y efectos de la implementación de una plataforma digital en el proceso de enseñanza de futuros docentes en matemáticas. Revista Virtual Universidad Católica del Norte, (57), 137-156. DOI: https://doi.org/10.35575/rvucn.n57a10

35. Prensky, M. (2010). Nativos e Inmigrantes Digitales. Cuadernos SEK 2.0.

36. Rodríguez, A. (2014). Elaboración de un Objeto Virtual de Aprendizaje (OVA), que integre el origami como facilitador de la enseñanza de los sólidos en Geometría (Trabajo de maestría). Universidad Nacional de Colombia, Bogotá, Colombia.

37. Rojas, C. (2015). Objetos virtuales de aprendizaje como herramienta para la enseñanza del álgebra en el grado octavo de la Institución Educativa Ana de Castrillón (Trabajo de maestría). Universidad Nacional de Colombia, Medellín, Colombia. 
38. Sarmiento, M. (2007). La enseñanza de las matemáticas y las NTIC. Una estrategia de formación permanente (Tesis doctoral). Universitat Rovira i Virgili, Tarragona, España.

39. Schneider, W. (1987), "Connectionism: it is a paradigm shift for psychology?”. Behavior Research Methods, Instruments, \& Computers, 19(2), 73-83. DOI: https://doi.org/10.3758/BF03203762

40. Secretaría de Educación del Distrito. (2014). Análisis descriptivo y pedagógico de los resultados de las pruebas Saber $3^{\circ}, 5^{\circ}$ y $9^{\circ}(2009,2012$ y 2013) y Saber 11 (2014). Bogotá, Colombia.

41. Tovar, I. (2004). Los Objetos Virtuales de Aprendizaje y su impacto en la calidad del proceso de la calidad del proceso de virtual. Revista de Tecnología de Información y Comunicación en Educación, 8(1), 113-126.

42. Triana, S. (2015). Diseño y construcción de un objeto virtual de aprendizaje para la asignatura de Ingeniería web (Trabajo de grado). Universidad Libre, Bogotá, Colombia.

43. Tupiza, L. (2018). Diseño de una guía docente para la enseñanza de matemática con el apoyo de las TIC (Trabajo de grado de maestría). Pontificia Universidad Católica del Ecuador, Quito, Ecuador.

44. Vygotsky, L. (1978). La mente en la sociedad: El desarrollo de los procesos psicológicos superiores. Cambridge, MA: Harvard University Press.

45. Zapata, C., Bonfante M., \& Suárez, M. (2013). Calidad de objetos virtuales de aprendizaje para el desarrollo de la inteligencia práctica en niños sordos. Hexágono pedagógico, 4(1), 1-26. DOI: https://doi.org/10.22519/2145 888X.307

46. Zapata, D., Estrada, E., \& Chaparro, L. (2015). Diseño y construcción de un objeto virtual de aprendizaje, como estrategia para fortalecer la comprensión del concepto de fracción en los estudiantes de grado octavo del Colegio Salucoop Sur IED (Trabajo de especialización). Fundación Universitaria Los Libertadores, Bogotá, Colombia.

[1] Artículo de investigación que se deriva del proyecto de Maestría en Educación Matemática, de la Universidad Francisco de Paula Santander, titulado "Objeto virtual de aprendizaje como apoyo para el desarrollo de las habilidades numéricas en estudiantes de educación básica”. 